\title{
EARLANDIACEA (FORAMINIFERIDA) DEL CARBONÍFERO INFERIOR DEL ÁREA DEL GUADIATO (SUROESTE DE ESPAÑA)
}

\author{
Pedro CÓZAR y Sergio RODRÍGUEZ \\ Departamento de Paleontología, Facultad de Ciencias Geológicas, Universidad \\ Complutense de Madrid, 28040-Madrid, E-mail: Sergrodr@eucmax.sim.ucm.es.
}

Cózar, P. y Rodríguez, S. 2000. Earlandiacea (Foraminiferida) del Carbonífero inferior del Área del Guadiato (Suroeste de España). [Earlandiacea (Foraminiferida) from the Lower Carboniferous of Guadiato Area (southwestern Spain)]. Revista Española de Paleontología, 15(1), 89-99. ISSN 0213-6937.

\begin{abstract}
The Earlandiacea from Guadiato Area are described in this contribution. Foraminiferal zones 14 to 18 , which are included in upper Viséan and Serpukhovian (lower Carboniferous), have been identified in that region. The identified species are: Earlandia minima (Birina), E. elegans (Rauser-Chernoussova and Reitlinger), E. moderata (Malakhova), E. clavatula (Howchin), E. vulgaris (Rauser-Chernoussova and Reitlinger), Pseudoammodiscus priscus (Rauser-Chernoussova and Reitlinger), P. parvus (Reitlinger), P. volgensis (Rauser-Chernoussova and Reitlinger), P. pulchrus (Malakhova), P. sp. A, Pseudoglomospira spp., Pseudolituotuba gravata (Conil and Lys).

In addition, Brunsia sp. and Volvotextularia sp., represented by few specimens are also figured. In spite of their scarcity they are important to know the whole assemblage. Furthermore, Volvotextularia sp. has been used to define a closer palaeobiogeographic relationship to Morocco basins, in spite of which the southern Iberian Peninsula usually is located closer to European basins.
\end{abstract}

Keywords: Earlandiidae, Pseudoammodiscidae, Pseudolituotubidae, upper Viséan, Serpukhovian, OssaMorena, Spain.

\section{RESUMEN}

En este trabajo se describen los Earlandiacea encontrados en los sedimentos del ¡rea del Guadiato. En estos materiales se han identificado las zonas 14 a 18 de foraminíferos, que pertenecen al Viseense superior y Serpukhoviense (Carbonífero inferior). Las especies descritas son: Earlandia minima (Birina), E. elegans (Rauser-Chernoussova y Reitlinger), E. moderata (Malakhova), E. clavatula (Howchin), E. vulgaris (RauserChernoussova y Reitlinger), Pseudoammodiscus priscus (Rauser-Chernoussova y Reitlinger), P. parvus (Reitlinger), P. volgensis (Rauser-Chernoussova y Reitlinger), P. pulchrus (Malakhova), P. sp. A, Pseudoglomospira spp., Pseudolituotuba gravata (Conil y Lys).

Además, se figuran las especies Brunsia sp. y Volvotextularia sp., de las que han sido encontrados muy pocos ejemplares, pero que son importantes para completar la asociación. Además, Volvotextularia sp. ha sido utilizada para definir una relación paleobiogeográfica próxima a las cuencas de Marruecos, a pesar de que en ocasiones se sit en estas cuencas del sur de la Península Ibérica más próximas a las cuencas europeas.

Palabras clave: Earlandiidae, Pseudoammodiscidae, Pseudolituotubidae, Viseense superior, Serpukhoviense, Ossa-Morena, España.

\section{INTRODUCCIÓN}

En el suroeste de la Península Ibérica los afloramientos del Carbonífero son frecuentes. Este trabajo está basado en parte de la asociación de foraminíferos encontrada en uno de estos afloramientos, en concreto en el Área del Guadiato (Fig. 1), compuesta por rocas tanto carbonatadas como siliciclásticas del Viseense superior y Serpukhoviense inferior.
Las rocas del Carbonífero inferior del Guadiato fueron dividas en tres unidades estructurales (Cózar y Rodríguez, 1999): U. del Fresnedoso, U. Sierra del Castillo y U. San Antonio-La Juliana. En las dos primeras unidades las zonas de foraminíferos identificadas son semejantes (Viseense superior), mientras que la U. San Antonio-La Juliana está formada por sedimentos del Serpukhoviense en los que están incluidos olistolitos del Viseense superior. El estudio y datación de los materiales 


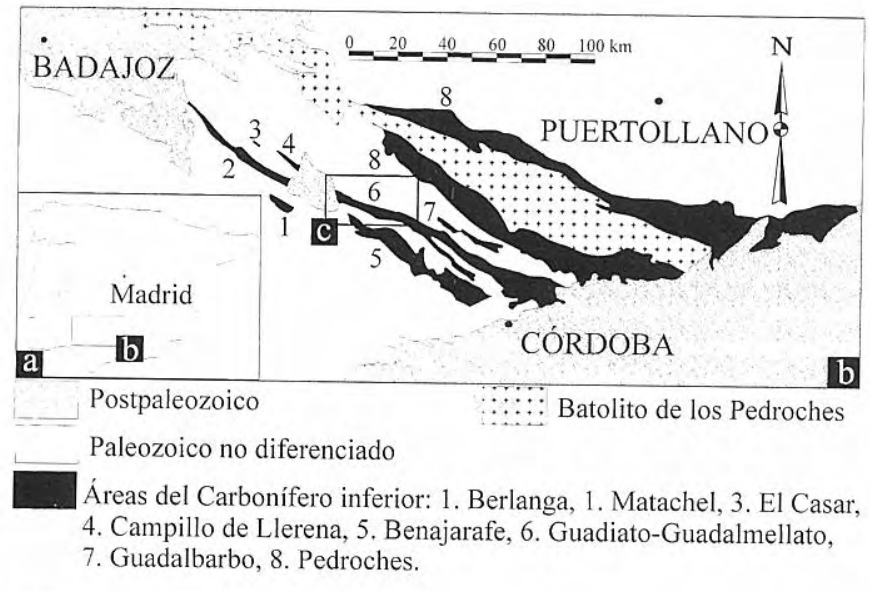

Figura 1. a: Península Ibérica. b: Principales afloramientos del Carbonífero inferior en la zona norte de OssaMorena. c: Área estudiada (modificado de Cózar y Rodríguez, 1999).

se ha centrado fundamentalmente sobre las rocas carbonatadas que afloran en las unidades mencionadas.

La datación de estos materiales está basada en las zonas de foraminíferos propuestas por Mamet (1974), de las que han sido identificadas desde la 14 hasta la 18. Aunque en este trabajo de Mamet no se expone claramente el tipo de zonas que son, después de un análisis detallado de las diferentes publicaciones del autor en las que se hace referencia a la zonación (Cózar Maldonado, 1996, 1998), se puede precisar que son cronozonas.

La diversidad de formas que desarrollaron los foraminíferos en este intervalo es muy alta. Entre todas las familias presentes, este trabajo se centra en Earlandiacea; esta superfamilia agrupa los foraminíferos más simples de toda la asociación, formados por una cámara tubular recta o enrollada, y con la pared formada por una única capa de naturaleza microgranular. En las listas de sinonimia solo se expondrán las citas de las descripciones originales y aquellas citas que se han considerado más interesantes y representativas.
Todas las láminas delgadas en las que está basado este estudio están depositadas en el Departamento de Paleontología de la Facultad de Ciencias Geológicas (Universidad Complutense de Madrid). Las siglas que aparecen en cada una de las figuras corresponden a las secciones estratigráficas, tramo de la sección y número de ejemplar. Las abreviaturas utilizadas son: BUJ (Burjadillo), CCS (Cantera del Castillo), COL (Collado), COR (Cornuda), NVC (Navacastillo), SPL1 (Sierra Palacios 1), SPL2 (Sierra Palacios 2), SSE1 (Sierra de la Estrella 1), y VCR (Viacrucis). La localización de estas secciones estratigráficas está expuesta en Cózar Maldonado (1998).

\section{SISTEMÁTICA}

\author{
ORDEN FORAMINIFERIDA d'Eichwald, 1830 \\ SUBORDEN FUSULININA Wedekind, 1937 \\ Superfamilia Earlandiacea Cummings, 1955 \\ Familia Earlandiidae Cummings, 1955 \\ Género Earlandia Plummer, 1930
}

\section{Diagnosis}

Concha libre compuesta por un prolóculo redondeado o subredondeado y una segunda cámara tubular alargada no septada. La pared es microgranular.

\section{Observaciones}

Este género tiene la peculiaridad de que es muy fácil distinguir las diferentes especies aunque tengamos cortes oblicuos, ya que los valores específicos de separación son muy claros y sencillos. Algunos autores, como Armstrong y Mamet (1977) y Pinard y Mamet (1998) (Tabla 1), trabajan con este taxón a nivel de grupo de especies, diferenciando de esta forma 5 grupos principales: $E$. minima (Birina), E. elegans (Rauser-Chernoussova y Reitlinger), E. moderata (Malakhova), E. clavatula (Howchin) [=E. minor (Rauser-Chernoussova)], y $E$. vulgaris (Rauser-Chernoussova y Reitlinger). Las principales diferencias entre las especies de tamaños

\begin{tabular}{|l|c|c|c|c|c|c|c|c|}
\hline \multirow{2}{*}{ Especie } & \multicolumn{3}{|c|}{ D. e. de la cámara tubular $(\mathrm{en} \mu \mathrm{m})$} & \multicolumn{4}{c|}{ E. p. (en $\mu \mathrm{m})$} \\
\cline { 2 - 9 } & $\mathbf{1}$ & $\mathbf{2}$ & $\mathbf{3}$ & $\mathbf{4}$ & $\mathbf{1}$ & $\mathbf{2}$ & $\mathbf{3}$ & $\mathbf{4}$ \\
\hline E. minima (Birina) & $\approx 30$ & $\approx 30$ & $25-45$ & $25-51$ & 5 & 5 & $5-8$ & $4-11$ \\
\hline E. elegans (Rauser y Reitlinger) & $50-100$ & $50-75$ & $50-90$ & $48-95$ & $10-20$ & $10-20$ & $10-20$ & $8-26$ \\
\hline E. moderata (Malakhova) & $\approx 120$ & $100-130$ & $100-160$ & $95-163$ & $15-30$ & $20-30$ & $15-30$ & $18-34$ \\
\hline E. clavatula (Howchin) & $\approx 200$ & $150-250$ & $160-260$ & $144-231$ & $40-50$ & $45-60$ & $40-50$ & $29-45$ \\
\hline E. vulgaris (Rauser y Reitlinger, 1936) & $\approx 300$ & $300-400$ & $300-400$ & $250-350$ & $<80$ & $70-80$ & $<80$ & $38-86$ \\
\hline
\end{tabular}

Tabla 1. Principales parámetros utilizados en Earlandia. 1: Modificado de Armstrong y Mamet (1977). 2: Modificado de Conil et al. (1979). 3: Modificado de Pinard y Mamet (1998). 4: Asociación del Guadiato. Los parámetros de las columnas 1 y 3 están referidos a grupos de especies, y las columnas 2 y 4 , a especies. Abreviaturas: D.e. diámetro externo, E.p. espesor de la pared. 
similares se pueden definir como dos; la primera es el tamaño relativo del prolóculo, pero dentro de la bibliografía, en los casos que se han separado dos especies, la variación del diámetro del prolóculo está dentro de la posible variabilidad de una de ellas; tal vez este criterio serviría para diferenciar entre formas macro y microsféricas. La segunda diferencia es la forma de la cámara alargada, que se asemeje más a un tubo (el diámetro es bastante constante a lo largo de la misma), o que se asemeje a un cono (el diámetro en la parte próxima al prolóculo es mucho menor que en la abertura); esta segunda sí creemos que es específicamente válida.

Los parámetros utilizados para la diferenciación de las especies del Guadiato también se recogen en la Tabla 1.

Las cinco especies descritas del género Earlandia tienen unos parámetros que se asemejan bastante a los propuestos por Armstrong y Mamet (1977), Conil et al. (1979) y Pinard y Mamet (1998) (Tabla 1), aunque existen pequeñas variaciones entre los diferentes autores y a su vez con los valores que se han encontrado en el Guadiato. Además, en estos últimos, los rangos de algunos parámetros se solapan [D.e. de Earlandia moderata $($ Malakhova $)=95-163 \mu \mathrm{m}$, y en E. clavatula $($ Howchin $)=$ 124-231 $\mu \mathrm{m}]$. Esto se debe a que no hemos considerado el diámetro externo de la cámara tubular y el espesor de la pared como dos parámetros independientes, sino totalmente relacionados. Todos los ejemplares con un diámetro externo menor a $160 \mu \mathrm{m}$ podrían considerarse como pertenecientes a $E$. moderata (Malakhova), pero alguno de estos ejemplares tiene un espesor de la pared muy grande $(38 \mu \mathrm{m})$, por lo que se han clasificado como E. clavatula (Howchin). Por el contrario, hay ejemplares de esta misma especie con $220 \mu \mathrm{m}$ de diámetro externo y sólo $30 \mu \mathrm{m}$ en la pared. En consecuencia, se ha considerado que sólo una combinación de ambos parámetros permite una correcta diferenciación de las especies.

\section{Earlandia minima (Birina, 1948) Lám. I, fig. 1}

1948 Hyperammina minima Birina, 155 y 159, lám. 2, figs. 7-8.

Material: 16 ejemplares.

\section{Descripción}

Concha formada por un prolóculo esférico poco saliente y una cámara tubular de reducidas dimensiones. Diámetro externo de la cámara tubular entre 25 y $51 \mu \mathrm{m}$; diámetro interno de 14-30 $\mu \mathrm{m}$. Pared microgranular sin poros, con un espesor de 4-11 $\mu \mathrm{m}$. Diámetro externo del prolóculo de 30 $\mu \mathrm{m}$ a $85 \mu \mathrm{m}$. Longitud máxima de la concha de $580 \mu \mathrm{m}$.

\section{Discusión}

Se diferencia del resto de especies descritas del género por sus reducidas dimensiones (Tabla 1). El diámetro del prolóculo es un parámetro muy variable, ya que dentro del material estudiado están encuadradas tanto la forma microsférica como la macrosférica.

\section{Distribución}

En el Guadiato se ha encontrado en la zona 15 de la U. Sierra del Castillo y en las zonas 15 y 17 de la U. de San Antonio-La Juliana.

\section{Earlandia elegans (Rauser-Chernoussova y Reitlinger en Rauser-Chernoussova et al., 1936)} Lám. I, fig. 2

1936 Hyperammina elegans Rauser-Chernoussova y Reitlinger en Rauser-Chernoussova et al., 256-257, fig. 191.

Material: 33 ejemplares.

\section{Descripción}

Concha formada por un prolóculo esférico apenas saliente y una cámara tubular ligeramente curvada. Diámetro externo de la cámara tubular entre 48 y $95 \mu \mathrm{m}$; diámetro interno de 15-66 $\mu \mathrm{m}$. Pared microgranular sin poros, con un espesor de 8-26 $\mu \mathrm{m}$. Diámetro externo del prolóculo de 59 $\mu \mathrm{m}$ a $148 \mu \mathrm{m}$. Longitud máxima de la concha de $1100 \mu \mathrm{m}$.

\section{Discusión}

Se diferencia de E. minima (Birina) por sus mayores dimensiones, tanto en el diámetro de la cámara tubular como en el espesor de la pared (Tabla 1).

\section{Distribución}

En el Guadiato se ha encontrado en la zona 15 de la Unidad del Fresnedoso, en la zona 15 de la U. Sierra del Castillo y en las zonas 14, 15, 16, 17 y 18 de la U. de San Antonio-La Juliana.

\section{Earlandia moderata (Malakhova, 1954) Lám. I, fig. 3}

1954 Hyperammina moderata Malakhova, 50, lám. 1, fig. 3.

Material: 29 ejemplares.

\section{Descripción}

Concha formada por un prolóculo ligeramente alargado poco saliente y una cámara tubular ligeramente curvada. Diámetro externo de la cámara tubular entre 95 y $163 \mu \mathrm{m}$; diámetro interno de 48-120 $\mu \mathrm{m}$. Pared microgranular sin poros, con un espesor de 18-34 $\mu \mathrm{m}$. Diámetro externo del prolóculo de $115 \mu \mathrm{m}$ a $165 \mu \mathrm{m}$. Longitud máxima de la concha de $1300 \mu \mathrm{m}$.

\section{Discusión}

Se diferencia de las dos especies anteriormente descritas por tener el diámetro de la cámara tubular y el espesor de la pared mayores (Tabla 1).

\section{Distribución}

En el Guadiato se ha encontrado en la zona 15 de la U. Sierra del Castillo y en las zonas 14, 15, 17 y 18 de la U. de San Antonio-La Juliana. 


\section{Earlandia clavatula (Howchin, 1888) Lám. I, fig. 5}

1888 Hyperammina elongata clavatula Howchin, 535, lám. 8 figs. 1-2.

1948a Hyperammina vulgaris minor Rauser-Chernoussova, 239, lám. 17, fig. 1.

1970 Earlandia vulgaris (Rauser-Chernoussova y Reitlinger); Hallett, 890, lám. 3, fig. 10.

Material: 25 ejemplares.

\section{Descripción}

Concha formada por un prolóculo esférico o algo ovalado y una cámara tubular ligeramente curvada. Diámetro

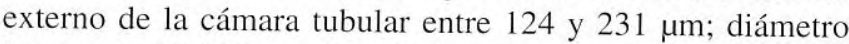
interno de 48-192 $\mu \mathrm{m}$. Pared microgranular sin poros, con un espesor de 29-45 $\mu \mathrm{m}$. Diámetro externo del prolóculo de 110 $\mu \mathrm{m}$ a $260 \mu \mathrm{m}$. Longitud máxima de la concha de $1600 \mu \mathrm{m}$.

\section{Discusión}

Se diferencia de las especies anteriormente descritas por sus mayores dimensiones (Tabla 1). En la bibliografía es más común que el taxón sea citado como Earlandia minor (Rauser-Chernoussova), pero éste tiene los mismos parámetros y características que E. clavatula (Howchin), que fue descrita previamente. La especie descrita por Hallett (1970) como E. vulgaris (Rauser-Chernoussova) también tiene parámetros semejantes a los de E. clavatula (Howchin).

\section{Distribución}

En el Guadiato se ha encontrado en la zona 15 de la Unidad del Fresnedoso, en la zona 15 de la U. Sierra del Castillo y en las zonas 14, 15, 17 y 18 de la U. de San Antonio-La Juliana.

\section{Earlandia vulgaris (Rauser-Chernoussova y Reitlinger, en Rauser-Chernoussova et al., 1936) \\ Lám. I, figs. 4 y 6}

1936 Hyperammina vulgaris Rauser-Chernoussova y Reitlinger, en Rauser-Chernoussova et al., 255-256, lám. 1, fig. 190.

1955 Earlandia pulchra Cummings, 228-229, lám. 1, figs. 1, 15-16.

Material: 15 ejemplares.

\section{Descripción}

Concha formada por un prolóculo alargado y saliente, y una segunda cámara tubular. Diámetro externo de la cámara tubular entre 250 y $350 \mu \mathrm{m}$; diámetro interno de 111-250 $\mu \mathrm{m}$. Pared microgranular sin poros, con un espesor de 38-86 $\mu \mathrm{m}$. Diámetro externo del prolóculo de $290 \mu \mathrm{m}$ a $375 \mu \mathrm{m}$. Longitud máxima de la concha de $2200 \mu \mathrm{m}$.

\section{Discusión}

Esta especie es la de mayor tamaño de todas las descritas (Tabla 1). La diferencia fundamental entre $E$. vulgaris y E. pulchra está en la relación entre el diámetro interno y el externo de la cámara tubular, siendo mayor en la segunda especie; esto significa que el espacio libre en el interior de la cámara tubular es menor. Consideramos esta diferencia insuficiente, por lo que $E$. pulchra se ha puesto en sinonimia con la especie $E$. vulgaris.

\section{Distribución}

En el Guadiato se ha encontrado en la zona 14 de la Unidad del Fresnedoso, en la zona 15 de la U. Sierra del Castillo y en las zonas 14 y 15 de la U. de San Antonio-La Juliana.

\section{Lámina I}

1 Earlandia minima (Birina). SPL2-2'-5, U. San AntonioLa Juliana, Zona 14 (x95).

2 Earlandia elegans (Rauser-Chernoussova y Reitlinger). VCR/7b'-8, U. San Antonio-La Juliana, Zona 17 (x65).

3 Earlandia moderata (Malakhova). COL/9h'-5, U. Sierra del Castillo, Zona 15 (x37).

4 Earlandia vulgaris (Rauser-Chernoussova y Reitlinger). SPL1/3ah'bis-3, U. San Antonio-La Juliana, Zona 14 (x25).

5 Earlandia clavatula (Howchin). COL/7ch-19, U. Sierra del Castillo, Zona 15 (x95).

6 Earlandia vulgaris (Rauser-Chernoussova y Reitlinger). COL/13h-13, U. Sierra del Castillo, Zona 15 (x37).

7 Pseudoammodiscus priscus (Rauser-Chernoussova). COR/9'bis-5, U. San Antonio-La Juliana, Zona 18 (x155).

8 Pseudoammodiscus priscus (Rauser-Chernoussova). BUJ 14' 'bis-9, U. San Antonio-La Juliana, Zona 15 (x240).

9 Pseudoammodiscus parvus (Reitlinger). SSE1/1-28, U. Sierra del Castillo, Zona 15 (x240).
10 Pseudoammodiscus parvus (Reitlinger), BUJ A-3, U. San Antonio-La Juliana, Zona 14 (x155).

11 Pseudoammodiscus pulchrus (Malakhova). COL/13h-12, U. Sierra del Castillo, Zona 15 (x120).

12 Pseudoammodiscus volgensis (Rauser-Chernoussova) CCS/14t-16, U. Sierra del Castillo, Zona 15 (x95).

13 Pseudoammodiscus pulchrus (Malakhova). SSE1/1h'-1, U. Sierra del Castillo, Zona 15 (x95).

14 Pseudoammodiscus sp. A. SPL1/5a'-5, U. San AntonioLa Juliana, Zona 15 (x95).

15 Pseudoammodiscus sp. A. NVC/4ah'-1, U. Sierra del Castillo, Zona 15 (x95).

16 Brunsia sp. CCS/8bh-14, U. Sierra del Castillo, Zona 15 (x155).

17 Pseudoglomospira sp., BUJ A'-7, U. San Antonio-La Juliana, Zona 18 (x65).

18 Volvotextularia sp., BUJ A'-13, U. San Antonio-La Juliana, Zona 18 (x240).

19 Pseudolituotuba gravata (Conil y Lys). BUJ 49-3, U. San Antonio-La Juliana, Zona 14 (x65). 
Lámina I
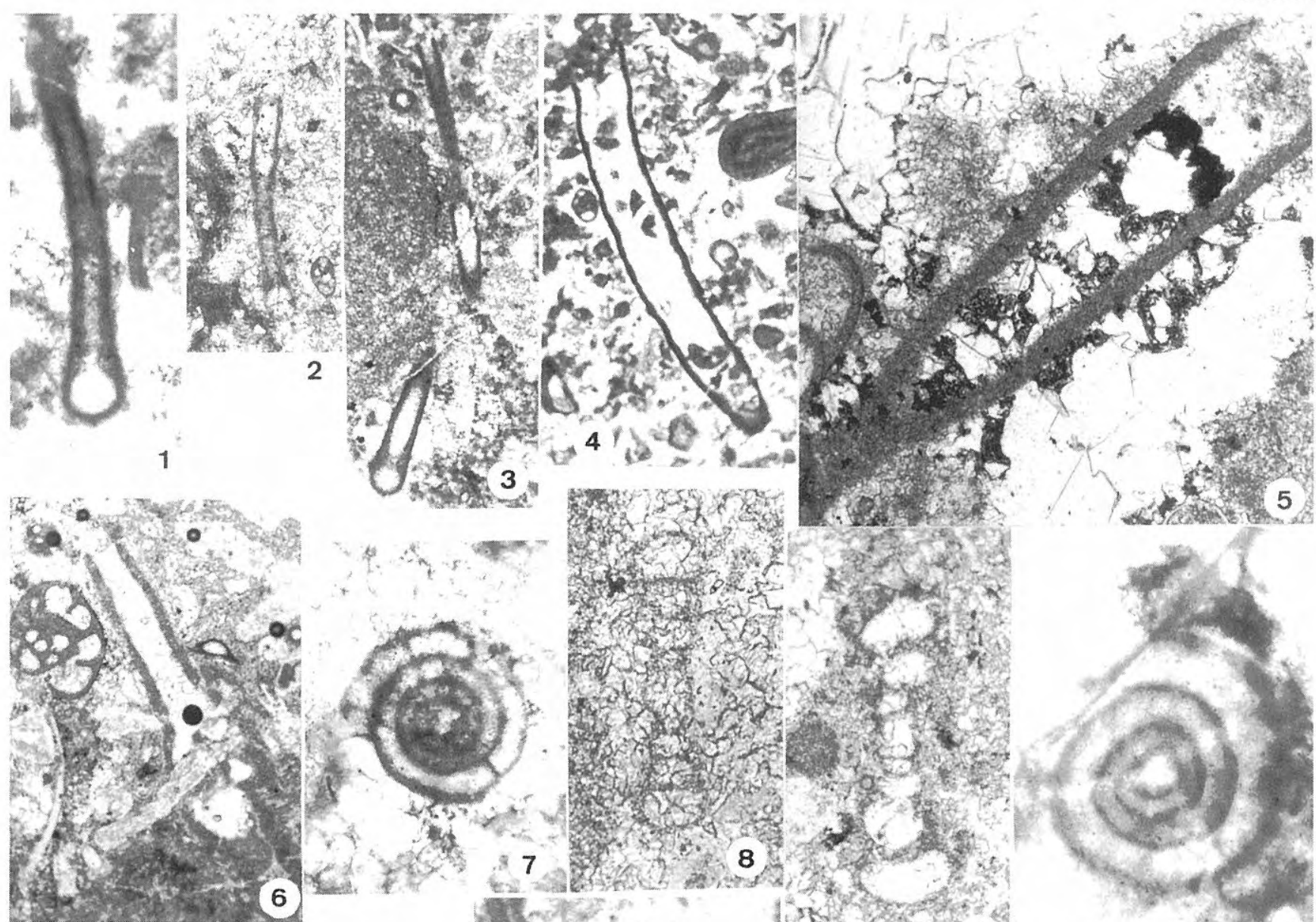

asom,

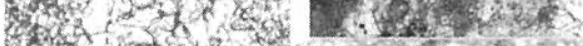

astancis

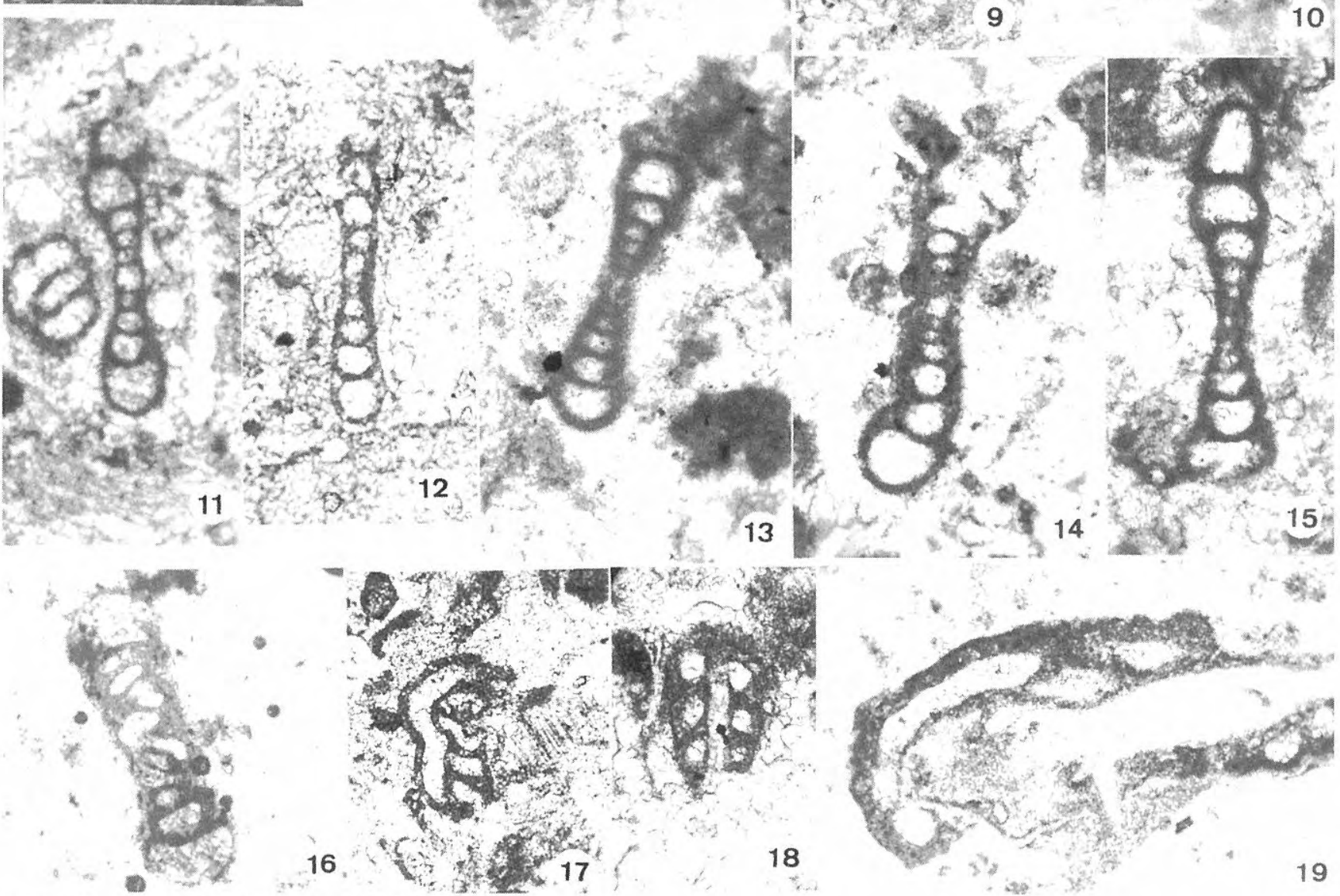

Revista Española de Paleontología, 15 (1), 2000.
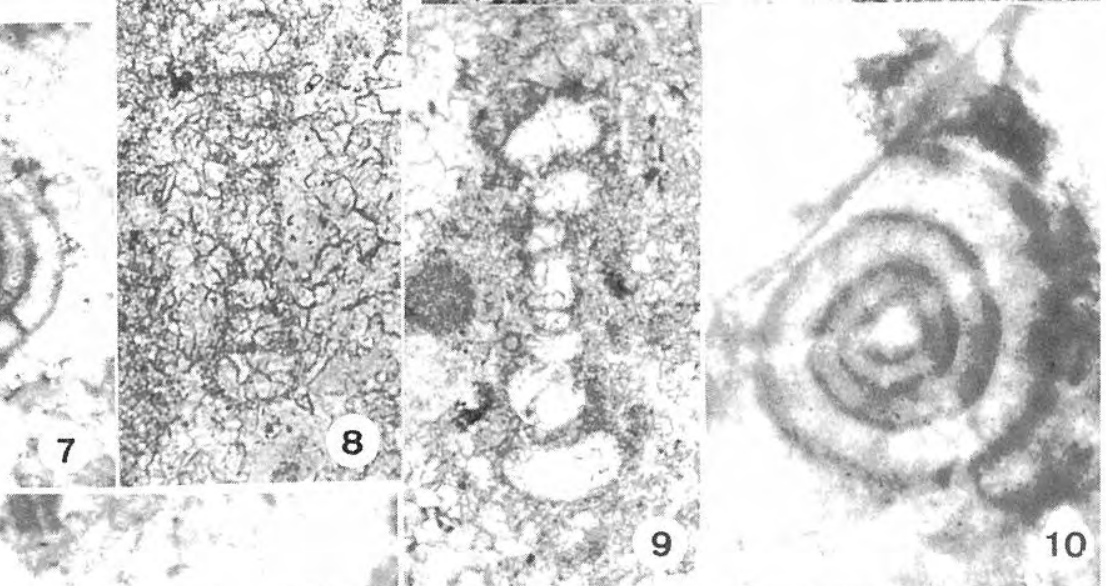


\begin{tabular}{|l|c|c|c|c|c|c|c|c|}
\hline \multirow{2}{*}{ Especie } & \multicolumn{2}{|c|}{ N. v. } & \multicolumn{2}{c|}{ D. c. $(\mathrm{en} \mu \mathrm{m})$} & \multicolumn{2}{c|}{ E.p. $(\mathrm{en} \boldsymbol{\mu m})$} & \multicolumn{2}{c|}{ D. i. p. $(\mathrm{en} \boldsymbol{\mu m})$} \\
\cline { 2 - 10 } & $\mathbf{1}$ & $\mathbf{2}$ & $\mathbf{1}$ & $\mathbf{2}$ & $\mathbf{1}$ & $\mathbf{2}$ & $\mathbf{1}$ & $\mathbf{2}$ \\
\hline P. priscus (Rauser) & 3 & $<4$ & $170-250$ & $125-280$ & $5-8$ & $5-10$ & 50 & $18-40$ \\
\hline P. parvus (Reitlinger) & Pocas & $<4$ & $190-320$ & $230-330$ & 12 & $14-18$ & $44-68$ & $35-67$ \\
\hline P. volgensis (Rauser) & 4,5 & $4-6$ & $\approx 400$ & $250-450$ & 15 & $12-25$ & $30-50$ & $12-27$ \\
\hline P. pulchrus (Malakhova) & $4-5$ & $4-6$ & 420 & $300-440$ & 17 & $10-20$ & 42 & $30-55$ \\
\hline P. sp A & & $3-6$ & & $530-770$ & & $16-22$ & & 30 \\
\hline
\end{tabular}

Tabla 2. Principales parámetros de las especies de Pseudoammodiscus. En las columnas 1 están los parámetros del material original de las distintas especies, y en las columnas 2 los de la asociación del Guadiato. Abreviaturas: N.v. número de vueltas, D.c. diámetro de la concha, E.p. espesor de la pared, D.i.p. diámetro interno del prolóculo.

\section{Familia Pseudoammodiscidae \\ Conil y Lys, en Conil y Pirlet, 1970}

\section{Diagnosis}

Concha libre, compuesta por un prolóculo redondeado y una cámara tubular con un enrollamiento que puede ser desde planoespiral a totalmente descentrado y desorganizado. La pared es microgranular.

\section{Observaciones}

Además de las especies de Pseudoammodiscus y Pseudoglomospira que se describen a continuación, también se han encontrado representantes de los géneros Brunsia y Volvotextularia, que no se describirán en detalle debido al escaso material del que se dispone.

De Brunsia sp. (Lám. I, fig. 16) hemos encontrado tres ejemplares (dos en sección ecuatorial y uno en sección axial), pero bastante mal conservados.

Volvotextularia sp. (Lám. I, fig. 18) también está muy poco representada. El género, en la bibliografía, es habitualmente confundido con Trepeilopsis, del que se diferencia por tener la pared microgranular.

\section{Género Pseudoammodiscus}

Conil y Lys, en Conil y Pirlet, 1970

\section{Diagnosis}

Cocha libre, compuesta por un prolóculo y una cámara tubular con enrollamiento planoespiral.

\section{Observaciones}

Hemos determinado cinco especies del género Pseudoammodiscus, cuyos principales caracteres diagnósticos pueden verse en la Tabla 2.

\section{Pseudoammodiscus priscus}

(Rauser-Chernoussova, 1948) Lám. I, figs. 7-8

1948b Ammodiscus priscus Rauser-Chernoussova, 227, lám. 15 , figs. 2-3.

Material: 16 ejemplares.

\section{Descripción}

Concha discoidal, formada por un prolóculo (diámetro interno de $18-40 \mu \mathrm{m}$ ) y una cámara tubular planoespiral ligeramente umbilicada, con 3 a 4 vueltas evolutas. Tasa de expansión moderada a baja. El diámetro de la concha varía entre 125 y $280 \mu \mathrm{m}$, y la anchura en la última vuelta, entre 48 y $55 \mu \mathrm{m}$. La relación anchura/diámetro oscila entre 0,2 y 0,3 . La altura del lumen en la última vuelta es de 15-35 $\mu \mathrm{m}$. Pared microgranular con un espesor de 4-9,5 $\mu \mathrm{m}$.

\section{Discusión}

Esta especie es la más pequeña del género, con la pared muy delgada, y prolóculo relativamente grande. Se diferencia de $P$. parvus (Reitlinger) por un espesor de la pared menor y un diámetro del prolóculo algo mayor (Tabla 2).

\section{Distribución}

En el Guadiato se ha encontrado en la zona 15 de la U. Sierra del Castillo y en las zonas $14,15,17$ y 18 de la U. de San Antonio-La Juliana.

\section{Pseudoammodiscus parvus (Reitlinger, 1950) Lám. I, figs. 9-10}

1950 Ammodiscus parvus Reitlinger, 14, lám. 2, fig. 4.

Material: 6 ejemplares.

\section{Descripción}

Concha discoidal, formada por un prolóculo (diámetro interno de 33-67 $\mu \mathrm{m}$ ) y una cámara tubular planoespiral ligeramente umbilicada, con 3 a 3,75 vueltas evolutas. Tasa de expansión moderada a baja. El diámetro de la concha varía entre 231 y $335 \mu \mathrm{m}$, y la anchura en la última vuelta es alrededor de $55 \mu \mathrm{m}$. La relación anchura/diámetro es 0,2 . La altura del lumen en la última vuelta es de $24-48 \mu \mathrm{m}$. Pared microgranular con un espesor de $14-18 \mu \mathrm{m}$.

\section{Discusión}

Se diferencia de P. nodus (Conil y Lys) por una tasa de expansión de la espiroteca mucho menor. 


\section{Distribución}

En el Guadiato se ha encontrado en la zona 14 de la Unidad del Fresnedoso, en la zona 15 de la U. Sierra del Castillo y en las zonas 14 y 18 de la U. de San Antonio-La Juliana.

\section{Pseudoammodiscus volgensis (Rauser-Chernoussova, 1948) Lám. I, fig. 12}

1948b Ammodiscus volgensis Rauser-Chernoussova, 240, lám. 17, figs. 7-8.

1956 Ammodiscus buskensis Brazhnikova, 23-24, lám. 1, figs. 13-15, 17.

Material: 32 ejemplares.

\section{Descripción}

Concha discoidal, formada por un prolóculo (diámetro interno de 12-27 $\mu \mathrm{m}$ ) y una cámara tubular planoespiral umbilicada, con 3,5 a 5,5 vueltas evolutas. Tasa de expansión moderada. El diámetro de la concha varía entre 250 y $450 \mu \mathrm{m}$, y la anchura en la última vuelta, entre 63-134 $\mu \mathrm{m}$. La relación anchura/diámetro es 0,2-0,34. La altura del lumen en la última vuelta es de 30-50 $\mu \mathrm{m}$. Pared microgranular con un espesor de 5-23 $\mu \mathrm{m}$.

\section{Discusión}

Pseudoammodiscus buskensis (Brazhnikova) se diferencia por un diámetro menor de la concha para el mismo número de vueltas, y el espesor de la pared, también algo menor. Esta especie se ha puesto en sinonimia con $P$. volgensis (Rauser-Chernoussova) ya que se consideran mínimas e insuficientes estas diferencias.

\section{Distribución}

En el Guadiato se ha encontrado en las zonas 14 y 15 de la Unidad del Fresnedoso y U. Sierra del Castillo y en las zonas 14, 15, 16, 17 y 18 de la U. de San Antonio-La Juliana.

\section{Pseudoammodiscus pulchrus (Malakhova, 1956) \\ Lám. I, figs. 11 y 13}

1956 Ammodiscus pulchrus Malakhova, 96, lám. I, fig. 9.

Material: 8 ejemplares.

\section{Descripción}

Concha discoidal, formada por un prolóculo (diámetro interno de $30-55 \mu \mathrm{m}$ ) y una cámara tubular planoespiral ligeramente umbilicada, con 4 a 5 vueltas evolutas. Tasa de expansión moderada. El diámetro de la concha varía entre 300 y $440 \mu \mathrm{m}$, y la anchura en la última vuelta es de 90-115 $\mu \mathrm{m}$. La relación anchura/diámetro es 0,24-0,31. La altura del lumen en la última vuelta es de 37-50 $\mu \mathrm{m}$. Pared microgranular con un espesor de 8-20 $\mu \mathrm{m}$.

\section{Discusión}

Según las diagnosis originales de Pseudoammodiscus volgensis (Rauser-Chernoussova) y $P$. pulchrus (Malakhova), sus parámetros son semejantes (Tabla 2), pero ésta última tiene un prolóculo de mayor tamaño, más sobresaliente en relación con el resto de dimensiones de la concha. Esta diferenciación es mínima, y se podría pensar que insuficiente, de manera que en el Guadiato no represente más que las formas microsférica y macrosférica de una única especie. Por el momento, vamos a mantener las dos especies independientemente, hasta que el número de ejemplares sea suficiente como para realizar un estudio estadístico adecuado de la posible variabilidad intraespecífica.

\section{Distribución}

En el Guadiato se ha encontrado en la zona 14 de la Unidad del Fresnedoso, en la zona 15 de la U. Sierra del Castillo y en las zonas 14 y 15 de la U. de San Antonio-La Juliana.

\section{Pseudoammodiscus sp. A Lám. I, figs. 14-15}

Material: 7 ejemplares.

\section{Descripción}

Concha discoidal, formada por un prolóculo (diámetro interno de $30 \mu \mathrm{m}$ ) y una cámara tubular planoespiral umbilicada, con 3 a 6 vueltas evolutas. Tasa de expansión moderada a rápida. El diámetro de la concha varía entre 530 y $770 \mu \mathrm{m}$, y la anchura en la última vuelta es de 115-192 $\mu \mathrm{m}$. La relación anchura/diámetro es 0,2-0,27. La altura del lumen en la última vuelta es de 63-95 $\mu \mathrm{m}$. Pared microgranular con un espesor de $16-22 \mu \mathrm{m}$.

\section{Discusión}

Se diferencia del resto de especies del género por un gran tamaño para un número de vueltas bajo. No se ha descrito como una especie nueva ya que los especímenes encontrados son muy pocos, y no permiten un análisis adecuado de la variabilidad de la especie.

\section{Distribución}

En el Guadiato se ha encontrado en la zona 15 de la U. Sierra del Castillo y U. de San Antonio-La Juliana.

\section{Género Pseudoglomospira Bykova, en Bykova y Polenova, 1955}

\section{Diagnosis}

Concha libre, formada por un prolóculo y una cámara tubular con un enrollamiento de glomoespiral a irregular. La pared es microgranular.

\section{Observaciones}

Mamet (1970) incluye el género dentro de la familia Cornuspiridae. Conil y Lys, en Conil y Pirlet (1970), describen la familia Pseudoammodiscidae, en la que incluyen el género Pseudoglomospira junto a 
Pseudoammodiscus, Brunsia y el nuevo género Warnantella [especie tipo Glomospira tortuosa (Conil y Lys)]. Definen el nuevo género como caracterizado por un enrollamiento no centrado o incompletamente centrado, con una cámara tubular con replegamientos tortuosos o en zigzag.

Conil y Lys (1977) incluyen el género Pseudoglomospira en Tournayellidae debido a su enrollamiento variable y la presencia eventual de pseudoseptos. Conil et al. (1979) utilizan el género Warnantella para incluir formas semejantes a Glomospira subquadrata Potievskaya y Vakartchouk, con el mismo tipo de enrollamiento descrito previamente. Rich (1980) hace una revisión de Pseudoglomospira, sin considerar válido Warnantella. Altiner (1981) y Sebbar (1986) ponen en sinonimia Warnantella con Pseudoglomospira. Skipp et al. (1985) incluyen los especímenes con este tipo de enrollamiento irregular o tortuoso en Palaeonubecularia ?. Laloux (1988) justifica la separación de Warnantella por el modo de enrollamiento tortuoso.

Perret (1993) realizó una revisión histórica de los nombres utilizados, adoptando finalmente el de Pseudoglomospira. Esta autora encuentra dos grupos, el primero caracterizado por conchas esféricas u ovoides (con enrollamiento terminal a menudo sigmoidal), de pequeña talla, parte apelotonada desarrollada y diámetro del tubo bastante pequeño (Pseudoglomospira ex gr. elegans Lipina), formas que, según Conil (en Perret, 1993) y Laloux (1988), deberían ser incluidas en Brunsia emend. Conil y Lys (1977). El segundo grupo es el caracterizado por conchas cúbicas o irregulares, pseudoseptos muy débilmente marcados, con gran variabilidad en el diámetro de la concha y cámara tubular (Pseudoglomospira ex gr. subquadrata Potievskaya y Vakartchouk), que otros autores incluyeron en Warnantella. Aparte de los grupos quedan algunos especímenes de forma ovoidea, con diámetro grande de la cámara tubular y la última vuelta con el eje de enrollamiento situado a $90^{\circ}$ de la posición que mantiene la vuelta precedente.

Perret (1993), tras reconocer en los niveles de la Caliza de Ardengost todas las posibles variaciones, incluso en el mismo nivel, concluye que posiblemente se trate de variaciones intraespecíficas, que, por la ley de prioridad, corresponderían a Pseudoglomospira gordialis irregularis (Rauser-Chernoussova) y Pseudoglomospira subquadrata (Potievskaya y Vakartchouk), para formas angulosas y "picudas", respectivamente.

En la diagnosis original de Warnantella, la presencia de pseudoseptos no está contemplada, ya que esta diagnosis está basada exclusivamente en el modo de enrollamiento. En la diagnosis original de Pseudoglomospira, tampoco se hace referencia a pseudoseptos. Nosotros consideramos Warnantella como un sinónimo de Pseudoglomospira, pero tal y como fueron definidos originalmente, sin pseudoseptos. Por otro lado quedan todas aquellas especies en las que se puede reconocer una ligera pseudoseptación. La lista de estas especies está recogida en Perret (1993). Nosotros consideramos que éstas deberían ser incluidas en Tournayellidae Dain,
Subfamilia Septabrunsiininae Bykova (no sensu Conil y Lys).

Brunsia y Pseudoglomospira tienen especies tipo con morfologías muy distintas; pero especímenes mal orientados en secciones ecuatoriales de Brunsia, con las últimas vueltas alineadas (no planoespirales), se pueden confundir con los especímenes de Pseudoglomospira con morfologías redondeadas.

\section{Pseudoglomospira spp. Lám. I, fig. 17}

Material: 16 ejemplares.

\section{Descripción}

Concha libre, con un prolóculo y una cámara tubular enrollada glomoespiral o irregularmente. Periferia de la cocha redondeada, ovoidea o cúbica. El número de vueltas puede ser elevado. Pared microgranular, con un espesor de 8-40 $\mu \mathrm{m}$. El diámetro de la concha varía entre 202 y 500 $\mu \mathrm{m}$. La altura del lumen oscila entre 22 y $76 \mu \mathrm{m}$. El diámetro del prolóculo es $26-40 \mu \mathrm{m}$. La pared es microgranular.

\section{Discusión}

Un pequeño grupo de estos ejemplares tiene una forma totalmente irregular, donde no se puede llegar a definir ningún tipo de enrollamiento. Este tipo de disposición de la cámara tubular no ha sido definido en ninguna especie del género, pero, debido a lo irregular del mismo, no podemos precisar si corresponde a cortes tangenciales. Por el contrario, la mayor parte de los ejemplares tienen conchas redondeadas y ovoideas, con un enrollamiento glomoespiral irregular, que pueden asimilarse al grupo de especies de Pseudoglomospira elegans (Lipina), definido por Perret (1993).

\section{Distribución}

En el Guadiato se ha encontrado en la zona 14 de la Unidad del Fresnedoso, en la zona 15 de la U. Sierra del Castillo y en las zonas 14, 15 y 18 de la U. de San AntonioLa Juliana.

\section{Familia Pseudolituotubidae Conil y Longerstaey, en Conil et al., 1979 \\ Género Pseudolituotuba Vdovenko, 1971}

\section{Diagnosis}

Concha incrustante, formada por un prolóculo y una cámara tubular enrollada irregularmente o desenrollada. La pared es microgranular con abundantes partículas aglutinadas.

\section{Observaciones}

En trabajos recientes, se pueden encontrar dos nombres genéricos que corresponden al mismo taxón, Pseudolituotuba Vdovenko, 1971 y Vostokovella Pronina, 1972. El primero de ellos tiene prioridad, aunque siguen citándose ambos (Loeblich y Tappan, 1988). 
Conil y Longerstaey, en Conil et al. (1979), incluyen cuatro especies en este género, Pseudolituotuba wilsoni Conil y Longerstaey, P. berwicki Conil y Longerstaey, $P$. gravata (Conil y Lys) y P. extensa Conil y Longerstaey. Establecen las diferencias entre ellas por el modo de fijación o enrollamiento, el tamaño de la pared y de los ejemplares. No consideramos que los tres criterios utilizados sean formalmente válidos, sino que esos rasgos representan tan solo variaciones en los diferentes estadios ontogenéticos por los que pasa el organismo. Por tanto, al considerar estas variaciones como intraespecíficas, hasta ahora, el género es monoespecífico. Hay que considerar que se trata de un organismo que habitualmente depende de otro elemento para su fijación, y por lo tanto, su desarrollo y morfología dependen fundamentalmente de este elemento de fijación, ya que no es lo mismo que en la zona interna encontremos un pequeño foraminífero (la morfología al menos en su parte inicial será apelotonada), a que encontremos briozoos o un alga (la cámara tubular tenderá a desenrollarse y fijarse al elemento).

\section{Pseudolituotuba gravata (Conil y Lys, 1965)} Lám. I, fig. 19

1965 Lituotuba ? gravata Conil y Lys, 502, lám. 1, figs. 2-3. 1972 Vostokovella neivaensis Pronina, 10, lám. 1, figs. 5-6. 1979 Pseudolituotuba berwicki Conil et al., 27, lám. 28, fig. 26. 1979 Pseudolituotuba extensa Conil et al.,27-28, lám.30, fig. 18. 1979 Pseudolituotuba wilsoni Conil et al.,28, lám. 14, figs.3-4.

Material: 25 ejemplares.

\section{Descripción}

Concha libre o incrustante, con un prolóculo grande (60$400 \mu \mathrm{m})$ y una segunda cámara tubular con enrollamiento glomoespiral irregular en las primeras fases y desenrollado al final. Diámetro máximo de la concha de 630 a $5180 \mu \mathrm{m}$. Cámara tubular amplia desde su origen, con un diámetro interno en la parte final de 85-481 $\mu \mathrm{m}$. Pared calcárea, netamente aglutinante, cuyo espesor es 30-481 $\mu \mathrm{m}$.

\section{Discusión}

Todas las especies descritas en el género están diferenciadas por alguna característica en el enrollamiento. En un organismo incrustante, como es Pseudolituotuba, no se ha considerado el enrollamiento como un carácter válido para la diferenciación de especies, $\mathrm{y}$, por tanto, se considera el género como monoespecífico, teniendo prioridad el nombre $P$. gravata (Conil y Lys).

En este estudio no se describen ni contabilizan muchos cortes tangenciales y parciales. Sin embargo, hay algunas secciones estratigráficas donde este foraminífero puede llegar a ser un elemento mayoritario de la microfacies en algunos niveles.

\section{Distribución}

En el Guadiato se ha encontrado en la zona 15 de la Unidad del Fresnedoso y U. Sierra del Castillo y en las zonas 14, 15 y 17 de la U. de San Antonio-La Juliana.

\section{CONSIDERACIONES PALEOBIOGEOGRÁFICAS Y BIOESTRATIGRÁFICAS}

Los géneros Earlandia, Pseudoammodiscus, Brunsia, Pseudoglomospira y Pseudolituotuba son muy comunes en las rocas del Viseense-Serpukhoviense, tanto en cuencas del Norte de África, como de Rusia, de América y de Europa Occidental.

Las especies incluidas en estos géneros son bastante comunes en estas cuencas; solamente Pseudoammodiscus parvus (Reitlinger) y P. pulchrus (Malakhova) han sido citadas exclusivamente en la Cuenca de Moscú y en los Urales, respectivamente. También cabe mencionar que la primera sólo ha sido encontrada en el Carbonífero medio. Consideramos que la ausencia de estas dos especies y la distribución estratigráfica anómala de la primera se deben más a un problema taxonómico que paleobiogeográfico, ya que creemos que los especímenes pertenecientes a estas especies han sido posiblemente descritos como Pseudoammodiscus priscus (Rauser-Chernoussova) y $P$. volgensis (Rauser-Chernoussova), respectivamente.

A diferencia de estos géneros (y sus especies), Volvotextularia no ha sido citado en las cuencas de Europa Occidental. Este taxón ha sido uno de los pocos que se han utilizado en Cózar Maldonado (1998) para inferir una relación paleobiogeográfica más próxima del Área del Guadiato con las cuencas del norte de África, en concreto con las existentes en Marruecos, que con las de Europa Occidental.

Las especies descritas no tienen un gran interés bioestratigráfico para el grado de precisión del esquema de cronozonas propuesto por Mamet (1974), de las que se han reconocido las zonas 14, 15, 16, 17 y 18 (Cózar Maldonado 1998). Los rangos de distribución de los taxones considerados en este trabajo son muy amplios, y con una primera aparición de los mismos no muy bien definida o conocida. En consecuencia, la identificación de estas cinco cronozonas está basada en otros representantes pertenecientes a las familias Endothyridae, Biseriamminidae, Lasiodiscidae, Fusulinidae y Archaediscidae.

El dato bioestratigráfico más importante que aportan los representantes de Earlandiacea es la aparición de Volvotextularia. En otros afloramientos ha sido citado habitualmente a partir del Serpukhoviense. Los pocos ejemplares encontrados en el Área del Guadiato coinciden en los sedimentos que corresponden a la Zona 18. Esto puede tener relevancia bioestratigráfica para Sierra Morena, pero, debido a la escasez de ejemplares, no se puede confirmar esto hasta que se realicen estudios más detallados en otras áreas de esta región.

\section{CONCLUSIONES}

Se han descrito los representantes de la superfamilia Earlandiacea encontrados en el Área del Guadiato (Fig. 1). En esta región se han identificado materiales 
carbonatados pertenecientes al Viseense superior y Serpukhoviense inferior.

Estas edades fueron identificadas fundamentalmente por una amplia asociación de foraminíferos pertenecientes a otras superfamilias de Fusulinina, reconociéndose las zonas de foraminíferos de Mamet (1974) desde la 14 hasta la 18. De todas las especies descritas y figuradas en este trabajo, solamente Volvotextularia sp. puede tener valor bioestratigráfico a nivel regional.

Las especies de Earlandia se diferencian bastante fácilmente por el incremento de las dimensiones de sus conchas (Tabla 1).

Pseudoammodiscus sp. A es un taxón endémico del Guadiato, que presenta unas dimensiones muy grandes para un reducido número de vueltas. No se ha considerado describirlo formalmente como una especie nueva ya que los especímenes encontrados son escasos por el momento.

La identificación de los ejemplares de Pseudoglomospira se ha dejado en nomenclatura abierta, ya que los criterios taxonómicos existentes para la diferenciación de especies no parecen los más adecuados, de manera que algunos autores tienden a trabajar con grupos de especies. Siguiendo esta metodología, la mayor parte de los ejemplares del Guadiato se incluyen en el grupo de Pseudoglomospira elegans (Lipina), definido por Perret (1993).

Pseudolituotuba presenta una problemática similar a Pseudoglomospira, ya que para la diferenciación de especies no se ha considerado la irregularidad en el enrollamiento de la cámara tubular, ya que al ser un organismo incrustante, la morfología final de la concha depende de elemento sobre el que se fije. En consecuencia, se ha considerado el género como monoespecífico, teniendo prioridad la especie $P$. gravata (Conil y Lys), que es la que se describió en primer lugar.

Desde un punto de vista paleobiogeográfico, sólo un taxón, Volvotextularia, ha sido citado en las cuencas de Marruecos y no en las de Europa Occidental. En consecuencia, el Área del Guadiato parece tener más afinidad con estas cuencas del Norte de África que con las europeas. Otros taxones no descritos en este trabajo, pertenecientes a la superfamilia Endothyracea, apoyan esta conclusión (Cózar Maldonado, 1998).

Pseudoammodiscus parvus (Reitlinger) y P. pulchrus (Malakhova) sólo han sido citadas previamente en la Cuenca de Moscú y los Urales. La primera de ellas sólo en el Carbonífero medio. Estas diferencias se han considerado como un problema taxonómico más que paleobiogeográfico, ya que se interpreta que los representantes de estas especies son habitualmente descritos como Pseudoammodiscus priscus (RauserChernoussova) y $P$. volgensis (Rauser-Chernoussova), respectivamente. Pseudoammodiscus volgensis (RauserChernoussova) y P. pulchrus (Malakhova) podrían corresponder a las formas microsférica y macrosférica de una única especie.

\section{AGRADECIMIENTOS}

La investigación se llevó a cabo gracias a las subvenciones de los proyectos de la DGYCIT PB91-0083 y PB96-0842. La revisión del manuscrito por las Doctoras E. Villa y M.F. Perret, ha aportado gran cantidad de comentarios constructivos que han mejorado notablemente el manuscrito final.

\section{BIBLIOGRAFÍA}

Altiner, D. 1981. Recherches stratigraphiques et micropaléontologiques dans le Taurus Oriental au NW de Pinarbasi (Turquie). Thèse Sciences, 1-450, (inédite).

Armstrong, A. K. and Mamet, B. L. 1977. Carboniferous microfacies, microfossils, and corals, Lisburne Group, Arctic Alaska. U.S. Geological Survey Professional Paper, 849, 1-144.

Birina, L. M. 1948. Nuevas especies de algas calcáreas y foraminíferos de las capas de transición DevónicoCarbonífero de la cuenca de Moscú [en ruso]. Sovetskaya Geologia, Sbornik 28, 154-159.

Brazhnikova, N. E. 1956. Foraminíferos. In: Fauna y flora del Carbonífero de la cuenca de Galicie-Volyn [en ruso] (Eds. N.E. Brazhnikova, A.M. Istchenko, I.A. Istchenko, E.O. Novik, \& P.L. Choulga). Trudy Instituta Geologicheskikh Nauk, Akademiya nauk Ukrainskoy SSR, Seriya Stratigrafii i Paleontologi, 10, 16-103.

Bykova, E. V. \& Polenova E. I. 1955. Foraminíferos, radiolarios y ostrácodos del periodo Devónico de la región Volga-Urales [en ruso]. Trudy Vsesoyznogo Neftyanogo Nauchno-Issledomvatelískogo Geologorazvedochnogo Instituta (VNIGRI), 87 (Mikrofauna SSSR, Sbornik 6), 1-141.

Conil, R. et Lys, M. 1965. Précisions complémentaires sur la micropaléontologie du Dinantien. Annales de la Société Géologique de Belgique, 88(3), 25-44.

Conil, R. et Lys, M. 1977. Les transgressions dinantiennes et leur influence sur la dispersion et l'évolution des foraminifères. Mémoires de l'Institut de Géologie de l'Université de Louvain, 29, 9-55.

Conil, R. et Pirlet, H. 1970. Le Calcaire Carbonifère du synclinorium de Dinant et le sommet du Famennien. Congrès et Colloques de l'Universite de Liège, 55, 5253.

Conil, R., Longerstaey, P. J. et Ramsbottom, W. H. C. 1979. Matériaux pour l'étude micropaléontologique du Dinantien de Grande-Bretagne. Mémoires de l'Institut de Géologie de l'Université de Louvain, 30, 1-187.

Cózar, P. y Rodríguez, S. 1999. Propuesta de nueva nomenclatura para las unidades del Carbonífero Inferior del Sector Norte del Área del Guadiato (Córdoba). Boletín Geológico y Minero, 110(3), 237-254.

Cózar Maldonado, P. 1996. Biozonas de foraminíferos de la Sierra del Castillo (banda central de la Cuenca del Guadiato, Córdoba). Coloquios de Paleontología, 48, 47 72.

Cózar Maldonado, P. 1998. Bioestratigrafía con foraminíferos del Carbonífero Inferior del sector Norte 
del Área del Guadiato (Córdoba). Tesis doctoral de la Facultad de Ciencias Geológicas, Universidad Complutense de Madrid, 1-590, (inédita).

Cummings, R. H. 1955. New genera of foraminifera from the British Lower Carboniferous. Washington Academy of Sciences Journal, 45(1), 1-8.

Eichwald, E. d'. 1830. Zoologia specialis, vol. 2, D. E. Eichwaldus Vinius, 1-323.

Hallett, D. 1970. Foraminifera and algae from the Yoredale "series" (Viséan-Namurian) of Northern England. Comptes Rendus, $6^{\circ}$ Congrès International de Stratigraphie et de Géologie du Carbonifère, 873-901.

Howchin, W. 1888. Aditions to the knowledge of the Carboniferous foraminifera. Royal Microscopical Society Journal, 2, 533-545.

Laloux, M. 1988. Foraminifères du Viséen Supérieur et du Namurien du Bassin Franco-belge. In: Vise and Namurian beds in Belgium. Centenary symposium, Guidebook. Bulletin de la Société belge de Géologie, 96(3), 205-220.

Loeblich, A. R. and Tappan, H. 1988. Foraminiferal genera and their classification. Van Nostrand Reinhold Company, New York, 1-970.

Malakhova, N. P. 1954. Foraminíferos del Carbonífero Inferior de las calizas silíceas de la vertiente oeste de los Urales [en ruso]. Byulletin'Moskovskogo Obshchestva Ispytateley Prirody, Otdel Geologicheskii 29(1), 49-60.

Malakhova, N. P. 1956. Foraminíferos de las rocas del Carbonífero de la vertiente oeste de los Urales centrales y del norte [en ruso]. Trudy Gorno-Geologicheskogo Instituta, Akademiya Nauk SSSR, Ural'skiy Filial 24(3), 72-155.

Mamet, B. L. 1970. Carbonate microfacies of the Windsor Group (Carboniferous), Nova Scotia and New Brunswick. Geological Survey of Canada, Paper 70-21, 1-121.

Mamet, B. L. 1974. Une zonation par foraminifères du Carbonifère inférieur de la Téthys occidentale. Comptes Rendus, $7^{\circ}$ Congrès International de Stratigraphie et de Géologie du Carbonifère, 3, 391-408.

Perret, M. F. 1993. Recherches micropaléontologiques et biostratigraphiques (Conodontes-Foraminifères) dans le Carbonifère Pyreneen. Actes du Laboratoire de Géologie Sédimentaire et Paléontologie de l'Université PaulSabatier Toulouse, Série 2, Mémoires, 21, 1-597.

Pinard, S. et Mamet, B. L.1998. Taxonomie de petits foraminifères du Carbonifère supérieur-Permien inférieur du bassin de Sverdrup, Arctique canadien. Palaeontographica Canadiana, 15, 1-225.

Plummer, H. J. 1930. Calcareous foraminifera in the
Brownwood shale near Bridgeport, Texas. Texas University Bulletin, 3019, 5-21.

Pronina, T. V. 1972. Nuevos representantes de Astrorhizida del Paleozoico medio de los Urales. In: Nuevas especies fósiles de plantas e invertebrados de SSSR [en ruso]. Akademiya Nauk SSSR, Nauchnyy Sovet po Probleme, Moscow, 10-11.

Rauser-Chernoussova, D. M. 1948a. Algunos foraminíferos nuevos del Carbonífero inferior del distrito de Syzram [en ruso]. Trudy Instituta Geologicheskikh Nauk, Akademiya Nauk SSSR, 62 (Seriya Geologicheskaya 19), 239-243.

Rauser-Chernoussova, D. M. 1948b. Algunas nuevas especies de foraminíferos de los sedimentos del Carbonífero Inferior de la Cuenca de Moscú [en ruso]. Trudy Instituta Geologicheskikh Nauk, Akademiya Nauk SSSR, 62 (Seriya Geologicheskaya 19), 227-238.

Rauser-Chernoussova, D. M., Beljaev, G. M. \& Reitlinger, E. A. 1936. Foraminíferos del Paleozoico superior de la región del Pechora (NO de los Urales) [en ruso]. Trudy Polyarnoy Komisii Akademiya Nauk SSSR, 28, 59-232.

Reitlinger, E. A. 1950. Foraminifères des depóts du Carbonifère Moyen de la Plate-forme Russe a l'exclusion de la famille des Fusulinidae. Travaux Institut de Géologie de l'Academie des Sciences de l'URSS, 126 (Seriya Geologicheskaya 47), 1-125. Traduction par Bureau, Institut Français du Pétrole, 1456.

Rich, M. 1980. Carboniferous Calcareous Foraminifera from Northeastern Alabama, South-Central Tennessee, and northwestern Georgia. Cushman Foundation for Foraminiferal Research, Special Publication 18, 1-62.

Sebbar, A. 1986. Foraminifères et algues calcaires du Carbonifère inférieur (Viséen supérieur, Serpukhovien) de coupes significatives du Bassin de Béchar (Sahara Sud-Oranais, Algérie). Utilisation paléobiologique. Thèse 3eme. Cycle, Paris Orsay, 1-151, (inédite).

Skipp, B., Baesemann, J. F. and Brenckle, P. L. 1985. A reference area for the Mississippian-Pennsylvanian (midCarboniferous) boundary in east-central Idaho, USA. Comptes Rendus $10^{\circ}$ Congrès International de Stratigraphie et Géologie du Carbonifère, Madrid, 1983, 4, 403-428.

Vdovenko, M. V. 1971. Nuevos géneros de foraminíferos de los depósitos viseenses [en ruso]. Dopovidi Akademii Nauk Ukraïnsíkoï RSR, Seriya B, Geologichni, Kimichni ta Bilogichni Nauk, 33(10), 877-879.

Wedekind, P. R. 1937. Einführung in die Grundlagen der historichen Geologie, Band II, Mikrosbiostratigraphie, Die Korallen- und Foraminiferenzeit. Ferdinand Enke Verlag, Stuttgart, 1-136. 